\title{
Potential Mediators between Fibromyalgia and C-Reactive protein: Results from a Large U.S. Community Survey
}

\author{
Termeh Feinberg ${ }^{1,2^{*}} \mathbb{D}$, Usha Sambamoorthi ${ }^{3}$, Christa Lilly ${ }^{4}$ and Kim Karen Innes ${ }^{2,5}$
}

\begin{abstract}
Background: Fibromyalgia, a potentially debilitating chronic pain syndrome of unknown etiology, may be characterized by inflammation. In this study, we investigated the relation of FMS to serum C-reactive protein (CRP) in a large population of adults (18+) and investigated the influence of other factors on this relationship, including BMI, comorbidities, as well as mood and sleep disturbance.
\end{abstract}

Methods: Participants were 52,535 Ohio Valley residents (Fibromyalgia $n=1125$ ). All participants completed a comprehensive health survey (2005-2006) part of the C8 Health Project; serum levels of CRP were obtained, as was history of Fibromyalgia physician diagnosis. Logistic and linear regressions were used for this cross-sectional analysis.

Results: Mean CRP was higher among participants reporting Fibromyalgia than those without ( $5.54 \pm 9.8$ vs.3.75 \pm 7 . $2 \mathrm{mg} / \mathrm{L}, p<.0001)$ ). CRP level showed a strong, positive association with FMS (unadjusted odds ratio (OR) for highest vs. lowest quartile $=2.5$ (Cl 2.1,3.0; p for trend <.0001)); adjustment for demographic and lifestyle factors attenuated but did not eliminate this association (AOR for highest vs. lowest quartile $=1.4(\mathrm{Cl} 1.1,1.6 ; \mathrm{p}$ for trend $<.0001)$ ). Further addition of body mass index (BMI) and comorbidities to the model markedly weakened this relationship (AORs, respectively, for highest vs lowest CRP quartile $=1.2(\mathrm{Cl} 1.0,1.4)$ and $1.1(\mathrm{Cl}$ 0.9,1.3). In contrast, inclusion of mood and sleep impairment only modestly reduced the adjusted risk estimate (AORs for highest vs. lowest quartile $=1.3(\mathrm{Cl} 1.1,1.5)$ for each)).

Conclusions: Findings from this large cross-sectional study indicate a significant positive cross-sectional association of Fibromyalgia to serum C-reactive protein may be explained, in part, by BMI and comorbidity. Prospective research is needed to confirm this, and clarify the potential mediating influence of obesity and comorbid conditions on this relationship.

Keywords: Fibromyalgia, C-reactive protein, Inflammation, Body mass index, Comorbidity, Mediator, Epidemiology

\section{Background}

Chronic pain, defined as ongoing or recurrent pain that extends beyond the usual course of acute illness or injury for at least three to six months, is debilitating and costly. Over $20 \%$ of adults experience chronic pain at some point in their lives [1]. Fibromyalgia syndrome (FMS) is a rheumatologic chronic pain syndrome affecting approximately

\footnotetext{
*Correspondence: TFeinberg@som.umaryland.edu;

TermehFeinbergPhD@gmail.com

'Department of Family and Community Medicine, Center for Integrative Medicine, University of Maryland School of Medicine, 520 W. Lombard St., East Hall, Baltimore, MD 21201-1603, USA

${ }^{2}$ Department of Epidemiology, West Virginia University School of Public

Health, P.O. Box 9190, Morgantown, WV 26506-9190, USA

Full list of author information is available at the end of the article
}

$0.5-5 \%$ of populations in developed countries [2], including $1.75 \%$ of those in the U.S. [3]. FMS is characterized by a constellation of somatic symptoms that are typically present in addition to widespread pain (e.g., fatigue, sleep disturbance, memory, and mood problems), for which no clear cause can be found [4]. FMS is typically accompanied by morning stiffness, and sensitivity to loud noises, bright lights, and temperature extremes; women with FMS often report painful menstrual periods [5]. Although FMS affects both sexes and people of all ages, the majority (80-90\%) have been Caucasian [5, 6] middle-aged women [5]. Family members of FMS patients are at a higher risk for FMS [7]; the cause is unknown. 
While the etiology of FMS remains poorly understood, the widespread pain of FMS is thought to reflect abnormal central nervous system sensory information processing, with altered function in pain pathways and neuroendocrine disturbance [4]; Inflammatory processes may also play a significant role in the pathogenesis of FMS [8]. Although several MicroRNAs have been associated with FMS and symptom severity [9], diagnosis of FMS remains challenging, and there are currently no definitive diagnostic laboratory tests for the disease [10].

C-reactive protein (CRP), previously considered a biomarker of underlying infection or tissue injury [11], is now also believed to reflect chronic systemic inflammation [12]. CRP is considered a reliable proinflammatory biomarker [11] and is often included as part of the diagnostic laboratory workup for many rheumatological conditions [11]. While CRP is often included in the diagnostic workup for FMS, the relationship of CRP to FMS has not been clearly established.

Inflammation, as a characteristic reaction of tissues to injury or disease marked by physical swelling, redness, heat, and pain upon clinical examination, is not a classical symptom of FMS [10]. Inflammatory cytokines promote the development of contralateral hyperalgesia (an extreme, exaggerated reaction to pain) and allodynia (central pain sensitization following painful, often repetitive stimulation) [13]. FMS is typically characterized by both [14], and most [15-28], but not all recent studies $[23,29]$ have suggested a possible link between systemic inflammation and FMS. Of these, five studies measured CRP [24, 26-28, 30]; four suggested a positive association between CRP and FMS [24, 26-28]. However, to date, only one large, cross-sectional, population-based study has examined the association of CRP to FMS [27]. The study excluded women, and combined FMS with other pain syndromes, rendering assessment of the specific relationship between CRP and FMS difficult.

Moreover, to our knowledge, no studies to date examining the association of CRP to FMS have investigated the potential mediating effects of sleep or mood disturbance, factors linked to both elevated CRP levels and FMS [31-34]. Few have assessed the influence of elevated $\mathrm{BMI}[33,35]$, comorbidities, and other correlates [24]. This large, population-based study will fill the gaps in our understanding of the potential influence(s) of mood, sleep, BMI, and comorbid conditions on the relationship between CRP and FMS.

\section{Methods}

In this study, we investigated the relation of serum CRP levels to FMS in a large population of US adults.

\section{Data source}

This cross-sectional study used data from the C8 Health Project, which was conducted as part of the settlement of a class-action lawsuit stemming from drinking water contamination by Perfluorooctanoic Acid (PFOA) released from the DuPont Washington Works Plant near Parkersburg, WV, USA [36]. Data collection was conducted in 2005-2006 on individuals living or working in 6 PFOA-contaminated public water districts in West Virginia (WV) and Ohio (including those exposed to contaminated private-well drinking water) since 1951; a total of 69,030 individuals participated in the study, including $81 \%$ of eligible adults [36]. Project data collection was administered by an independent company, Brookmar, Inc. (Parkersburg, WV), and was conducted under the authority and supervision of the Wood County, WV, Circuit Court. Participants completed a comprehensive health questionnaire and volunteered a blood sample after completing individual consent forms for both. Demographic data and health survey completion were verified by trained project staff [36]. Project procedures, blood processing and assay methods, along with quality-assurance measures, have been described in detail elsewhere [36]. Briefly, following collection of each blood sample, serum was separated from red blood cells into single-use aliquots by centrifusion, and was refrigerated at individual data collection sites until daily pickup from a large, independent, accredited clinical diagnostic laboratory (LabCorp, Inc., Burlington, NC, USA). Samples were transported to a regional processing center (LabCorp, Inc., Columbus, OH) where they underwent analysis by latex immunoturbidimetry on a COBAS Integra 800 (Roche, Germany). The West Virginia University (WVU) IRB permitted access to the de-identified data by WVU investigators. Demographic, lifestyle, and health characteristics were determined via self-report; diagnoses of certain disorders, including diabetes and cardiovascular diseases, were further verified via chart review.

\section{Study population}

Our analysis excluded participants who were missing data on age or $<18$ years of age $(n=12,471,18.1 \%)$, pregnant $(n=640,1.1 \%)$; those reporting a cancer diagnosis and receiving treatment for diagnosed cancer other than non-melanoma skin cancer $(n=437,0.77 \%)$ to eliminate potential bias introduced by varied CRP levels as a result of chemotherapy treatment; those with service-related disabilities $(n=710,1.3 \%)$; and those who did not complete both the survey and blood work ( $n=468,0.83 \%)$. Participants with extreme body mass index (BMI) values $(<10.5$ and $>60.0)$ were also excluded to eliminate potential information bias $(n=94,0.17 \%)$. Further exclusion of persons with missing data on CRP 
and FMS $(n=40,0.002 \%)$ and other covariates of interest $(n=1673,3.1 \%)$, with the exception of covariates for which missing data on $>10 \%$ of participants occurred (for which a 'missing' category was included in analysis), yielded a final study sample of 52,535, including 51,410 FMS-free controls and 1125 adults with FMS (Fig. 1).

\section{Outcome variable}

Our primary, dichotomous outcome variable was FMS diagnosis, which was ascertained via self-report response to the question "Have you ever been diagnosed with Fibromyalgia?"

\section{Exposure variable}

Our primary exposure variable was serum level of inflammatory marker CRP $(\mathrm{mg} / \mathrm{L})$. The normal CRP range in the general population is considered $0.0-5.0 \mathrm{mg} / \mathrm{L}$ [28]. Further, in healthy young adult volunteer blood donors, the median concentration of CRP is $0.8 \mathrm{mg} / \mathrm{L}$, the 90th centile is $3.0 \mathrm{mg} / \mathrm{L}$, and the 99th centile is $10 \mathrm{mg} / \mathrm{L}$; following an acute-phase stimulus, values may increase from less than $50 \mu \mathrm{g} / \mathrm{L}$ to more than $500 \mathrm{mg} / \mathrm{L}$ [11]. C8

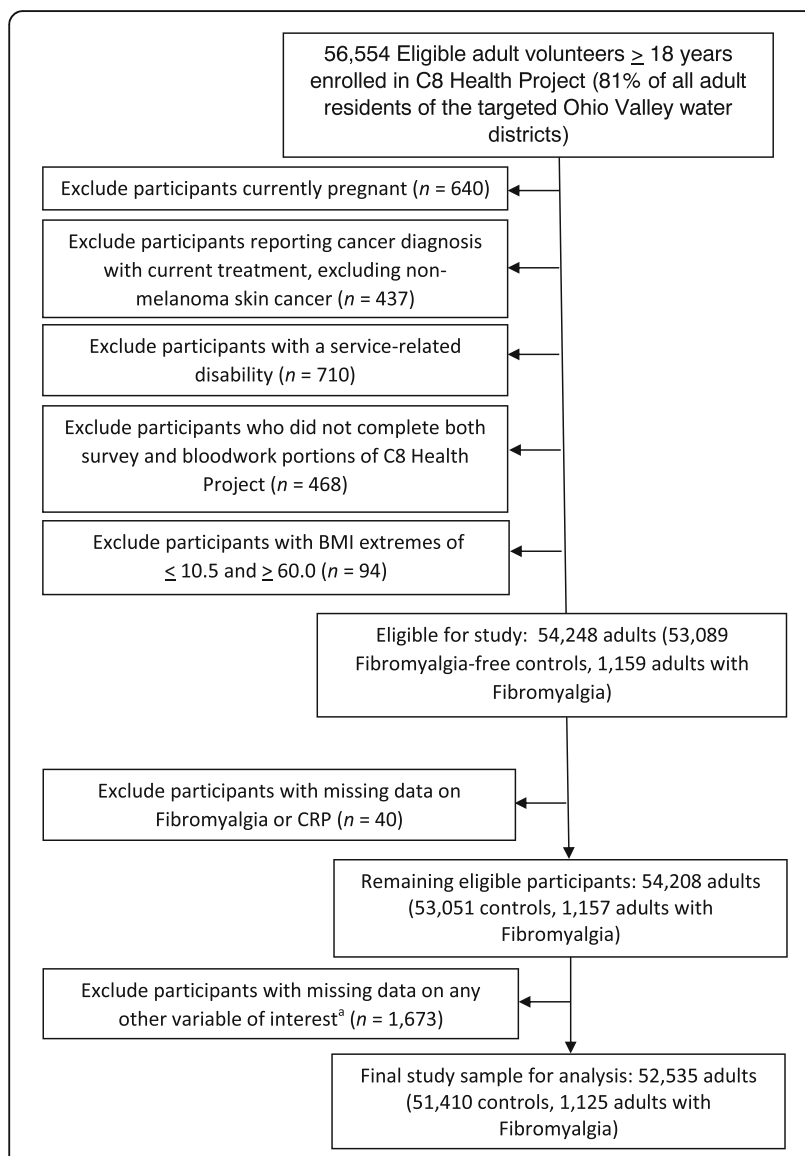

Fig. $1^{a}$ : with exceptions of income and current menstruation, which contained $\geq 10 \%$ of missing values; these were considered separate categories for analysis
Health Project coordinators recoded CRP values below the level of detection to $50 \%$ of the lowest level (i.e., " $<0.3$ " $\rightarrow 0.15)$. Likewise, values above the level of detection were recoded to $50 \%$ above the maximum level.

\section{Covariates}

In addition to age, gender, race, education, employment, marital status, income, alcohol or tobacco use, and exercise program status, comorbid conditions were also selected a priori as covariates if known or suspected to be associated with FMS and/or CRP. These included BMI [37], autoimmune conditions [38], osteoarthritis [39], kidney [40], respiratory [41], cardiovascular [40], liver [42], and endocrine [40] disease, diabetes [43], severe allergies [40] and sinus disease [44], stomach conditions [40, 42], and headache [45]. PFOA serum level and reproductive factors [46] were also examined as covariates.

\section{Potential mediating and modifying factors}

Potential mediating and modifying factors included fatigue, sleep impairment [42], mood disturbance [32, 40, 42], age [5], gender [5], obesity [35], tobacco use [47], statin use [48], and reproductive factors [46].

\section{Statistical analysis}

We conducted complete-case analyses using SAS 9.4 (Cary, NC, USA). Logistic regression analyses were used to evaluate the independent association of CRP level to FMS status, and to assess the influence of potential confounding, mediating, and modifying factors. The primary explanatory variable of interest, CRP, was analyzed as a continuous and categorical variable (study population quartiles, with the lowest percentile group used as the referent). Linear trends for CRP quartiles were assessed using polynomial contrasts. Differences between participants missing any data were assessed using logistic regression. Cox-Snell $\mathrm{R}^{2}$ values measured the predictive power of models. All $p$-values shown are two-sided.

All demographic characteristics, in addition to lifestyle factors significantly differing by FMS, were controlled for in multivariate models. BMI was categorized using the National Institutes of Health clinical classifications (scores of $\angle 25=$ 'Underweight or Normal weight'; 25$29.9=$ 'Overweight'; 30-34.9 = 'Obese Class 1'; and, $35+={ }^{\prime}$ Obese Classes 2/3') [49].

We evaluated the influence of both specific comorbid conditions and total number of comorbid conditions on the relation of CRP to FMS. A comorbidity index was created based on number of chronic comorbid conditions reported; these included: autoimmune disorders (defined as having any diagnoses of immune disease, lupus, or rheumatoid arthritis), osteoarthritis, allergies or frequent sinusitis, kidney disease, respiratory conditions (asthma, emphysema, chronic obstructive pulmonary disorder, 
bronchitis), heart disease, liver disease, endocrine disorders (thyroid, Addison's, or Cushing's disease), diabetes, or frequent headaches. This index was evaluated as both a continuous and categorical variable ('none,' 1 comorbidity', 2 comorbidities', and ' $3+$ comorbidities').

We assessed potential mediating influences of sleep impairment and mood disturbance. A composite sleep quality variable, with higher scores indicating poorer sleep quality, was derived from responses to individual questions regarding the frequency of short sleep, fitful sleep, insomnia, and/or daytime somnolence (scored as follows: 3 = 'frequently', 2 = 'sometimes', 1 = 'rarely', and 0 = 'never', for each). Mood disturbance was also assessed as a composite variable from responses to four individual questions, with higher scores indicating frequent mood swings, irritability, fatigue, and/or inability to concentrate (where 3 = 'frequently', 2 = 'sometimes', $1=$ 'rarely', and $0=$ 'never').

Additionally, we assessed the potential modifying influence of age ( $<45 \mathrm{vs.} \geq 45$ years), tobacco use ('current'/ 'not currently using tobacco'), gender, obesity (BMI $<30$ vs. $\geq 30$ ) current menstruation (yes/no), menopausal status (peri- or post-menopausal/premenopausal), statin use (yes/no), and sleep impairment and mood disturbance (scores of $<6$ vs. $\geq 6$, respectively) on the association between CRP and FMS.

We also conducted separate ancillary analyses adjusting for statin use (yes/no), PFOA serum level $(\mathrm{ng} / \mathrm{mL}$, evaluated as quartiles), and reproductive characteristics (women only) to determine the potential confounding influence of these factors on any observed association between CRP and FMS. Reproductive factors assessed included menopausal status ('pre-menopausal'; 'peri-or post-menopausal'; or 'unsure'), parity (number of pregnancies), and age at menarche ('16+ years of age'/'other age or unsure').

The first regression model assessed the crude association of CRP levels to reported FMS diagnosis. The second model was adjusted for demographic and lifestyle covariates, while additional models adjusted for BMI and comorbid conditions, as well as sleep and mood disturbance (separately and combined, respectively). We conducted additional analyses to evaluate the potential confounding influence of statin use, PFOA serum level, and (in female participants) reproductive history (i.e., menopausal status, age at menarche, and parity).

The potential mediating effects of BMI and comorbid conditions, as well as sleep impairment and mood disturbance were examined using separate logistic regression models. We also conducted ancillary analyses excluding autoimmune disorders rheumatoid arthritis, lupus, and self-reported 'previous diagnosis of immune disorder.' To evaluate the potential modifying effects of age, smoking, gender, obesity, current menstruation, menopausal status, statin use, sleep impairment, and mood disturbance, we conducted multivariable analyses; interactions were assessed by including the corresponding multiplicativeinteraction term in the statistical models.

\section{Results}

Relative to participants with complete data, those with missing data on key covariates had a higher number of comorbidities and were more likely to be poorly educated or to be retired, disabled, or unemployed ( $p$ 's $\leq$.002), but did not differ in other factors. Demographic and lifestyle characteristics are displayed in Table 1. Study participants were predominantly white (97.2\%), female (52.4\%), and married or cohabitating (68.9\%). Participant age ranged from 18.0 to 105.2 years, averaging $45.3(\mathrm{SD}=16.1)$ years. Most participants were employed or students $(64.1 \%)$ and overweight $(69.0 \%$ $\mathrm{BMI} \geq 25$, mean $\mathrm{BMI}=28.5, \mathrm{SD}=6.3$ ), while nearly half did not currently consume alcohol $(51.1 \%)$ and never smoked (43.5\%). CRP serum levels varied from 0.15 to 250.6 (mean CRP level $=3.79, \mathrm{SD}=7.2$ ) $\mathrm{mg} / \mathrm{L}$. Additionally, PFOA was not significantly associated with CRP after adjustment with all factors used in other models $(p=.084)$.

FMS was present in $2.1 \%$ of the study population $(n=1125)$. After adjustment for demographic and lifestyle factors, the odds of FMS increased $2 \%$ for every year-unit increase in age (Adjusted odds ratio $(\mathrm{AOR})=1.02,95 \%$ confidence interval $(\mathrm{CI}): 1.02,1.03$ (Table 1)), with a significantly higher mean age among FMS cases $(M=51.1, \mathrm{SD}=11.6)$ compared to controls $(M=45.1, \mathrm{SD}=16.2)$. Those aged $45-64$ years were 8.3 times more likely $(\mathrm{AOR}=8.28, \mathrm{CI} 4.91,13.95)$ than those in the age group 18-24 years to have FMS. Those with FMS were nearly 11 times more likely to be female $(\mathrm{AOR}=10.68, \mathrm{CI} 8.53,13.37)$. Participants who were employed or students were less likely to have FMS than all others, as were those with $<12$ th grade education, compared to those with higher educational attainment. Relative to participants who were married or cohabitating, those who were single were almost $40 \%$ less likely to report a diagnosis of FMS (AOR $=0.63$, CI 0.47, 0.84). Participants who reported current consumption of alcohol were slightly less likely to report a diagnosis of FMS ( $35.8 \%$ vs. $49.2 \%$, respectively; AOR $=0.86$, CI $0.75,0.98$ ), and women who had experienced menopause were about $80 \%$ more likely to have an FMS diagnosis compared to pre-menopausal women $(\mathrm{AOR}=1.82$, CI 1.49, 2.21).

Health characteristics of the study population are detailed in Table 2. Mean BMI was significantly higher among FMS cases $(M=30.47, \mathrm{SD}=7.2)$ than controls $(M=28.49, \mathrm{SD}=6.3)$, and was significantly and positively associated with FMS after adjustment for 
Table 1 Characteristics of a US adult population by Fibromyalgia (FMS) status, Adjusted for Demographic and Lifestyle factors, C8 Health Project, 2005-2006

\begin{tabular}{|c|c|c|c|c|}
\hline \multirow[t]{2}{*}{ Characteristic } & Total Population & FMS & Non-FMS & \multirow{2}{*}{$\begin{array}{l}\text { Adjusted Odds Ratio } \\
\text { OR }(\mathrm{Cl}), p \text {-value }\end{array}$} \\
\hline & \multicolumn{3}{|l|}{ N (\%) / Mean (SD) } & \\
\hline Total & 52,535 & $1125(2.1 \%)$ & $51,410(97.9 \%)$ & - \\
\hline Age (years) & $45.25(16.14)$ & $51.14(11.58)$ & $45.12(16.20)$ & $1.02(1.02-1.03),<.0001^{*}$ \\
\hline \multicolumn{5}{|l|}{ Age } \\
\hline 18-24 (ref) & $6540(12.5 \%)$ & $16(1.4 \%)$ & $6524(12.7 \%)$ & - \\
\hline $25-44$ & $20,178(38.4 \%)$ & $300(26.7 \%)$ & $19,879(38.7 \%)$ & $3.89(2.30-6.56),<.0001^{*}$ \\
\hline $45-64$ & $19,098(36.4 \%)$ & $689(61.2 \%)$ & 18,409 (35.8\%) & $8.28(4.91-13.95),<.0001^{*}$ \\
\hline $65+$ & 6719 (12.8\%) & $120(10.7 \%)$ & 6599 (12.8\%) & $4.37(2.50-7.64),<.0001^{*}$ \\
\hline \multicolumn{5}{|l|}{ Gender } \\
\hline Male (ref) & $25,002(47.6 \%)$ & $90(8.0 \%)$ & $24,912(48.5 \%)$ & - \\
\hline Female & $27,533(52.4 \%)$ & 1035 (92.0\%) & $26,498(51.5 \%)$ & $10.68(8.53-13.37),<.0001^{*}$ \\
\hline \multicolumn{5}{|l|}{ Race/Ethnicity } \\
\hline Non-Hispanic White & $51,092(97.2 \%)$ & $1098(97.6 \%)$ & $49,994(97.2 \%)$ & $1.09(0.73-1.62), .683$ \\
\hline Other Race (ref) & $1443(2.8 \%)$ & $27(2.4 \%)$ & $1416(2.8 \%)$ & - \\
\hline \multicolumn{5}{|l|}{ Education } \\
\hline$<12$ th grade (ref) & $6261(11.9 \%)$ & $106(9.4 \%)$ & 6155 (12.0\%) & - \\
\hline HS/GED & $22,353(42.6 \%)$ & $445(39.6 \%)$ & $21,908(42.6 \%)$ & $1.57(1.25-1.96), .0001^{*}$ \\
\hline Some College & $17,081(32.5 \%)$ & $442(39.3 \%)$ & $16,639(32.4 \%)$ & $2.22(1.75-2.82),<.0001^{*}$ \\
\hline Bachelor's degree+ & $6840(13.0 \%)$ & $132(11.7 \%)$ & $6708(13.1 \%)$ & $1.91(1.42-2.56),<.0001^{*}$ \\
\hline \multicolumn{5}{|l|}{ Employment } \\
\hline Employed/Student (ref) & $33,682(64.1 \%)$ & $482(42.8 \%)$ & $33,200(64.6 \%)$ & - \\
\hline Retired/Unemployed & 9057 (17.2\%) & $144(12.8 \%)$ & 8913 (17.3\%) & $1.44(1.16-1.79), .0008^{*}$ \\
\hline Disabled & $3357(6.4 \%)$ & $252(22.4)$ & $3105(6.0 \%)$ & $5.70(4.73-6.87),<.0001^{*}$ \\
\hline Homemaker & 5979 (11.4\%) & $233(20.7 \%)$ & $5746(11.2 \%)$ & $1.50(1.26-1.78),<.0001^{*}$ \\
\hline Other & $460(0.88 \%)$ & $14(1.2 \%)$ & $446(0.9 \%)$ & $2.23(1.28-3.88), .004^{*}$ \\
\hline \multicolumn{5}{|l|}{ Marital Status } \\
\hline Married/Cohabitating (ref) & $36,198(68.9 \%)$ & $836(74.3 \%)$ & $35,362(68.8 \%)$ & - \\
\hline Single & $8729(16.6 \%)$ & $60(5.3 \%)$ & 8669 (16.9\%) & $0.63(0.47-0.84), .002^{*}$ \\
\hline Divorced/Sep/Widow & 7608 (14.5\%) & $229(20.4 \%)$ & 7379 (14.4\%) & $0.85(0.71-1.01), .058$ \\
\hline \multicolumn{5}{|l|}{ Household Income } \\
\hline Don't know/Missing & 5335 (10.2\%) & $92(8.2 \%)$ & $5243(10.2 \%)$ & $0.85(0.66-1.10), .217$ \\
\hline$<\$ 20,000$ (ref) & $12,308(23.4 \%)$ & $288(25.6 \%)$ & $12,020(23.4 \%)$ & - \\
\hline$\$ 20,000-40,000$ & $14,326(27.3 \%)$ & $301(26.8 \%)$ & $14,025(27.3 \%)$ & $1.03(0.86-1.24), .759$ \\
\hline$\$ 40,001-70,000$ & $13,559(25.8 \%)$ & $291(25.9 \%)$ & $13,268(25.8 \%)$ & $1.05(0.86-1.29), .619$ \\
\hline$\$ 70,000+$ & 7007 (13.3\%) & $153(13.6 \%)$ & $6854(13.3 \%)$ & 1.09 (0.85-1.39), .514 \\
\hline \multicolumn{5}{|l|}{ Alcohol } \\
\hline Don't drink (ref) & $26,847(51.1 \%)$ & $722(64.2 \%)$ & $26,125(50.8 \%)$ & - \\
\hline Currently Drink & 25,688 (48.9\%) & $403(35.8 \%)$ & $25,285(49.2 \%)$ & $0.86(0.75-0.98), .023^{*}$ \\
\hline \multicolumn{5}{|l|}{ Tobacco User } \\
\hline Never (ref) & $22,859(43.5 \%)$ & 567 (50.4\%) & 22,292 (43.4\%) & - \\
\hline Current & 16,691 (31.8\%) & $274(24.4 \%)$ & 16,417 (31.9\%) & $0.91(0.78-1.07), .243$ \\
\hline
\end{tabular}


Table 1 Characteristics of a US adult population by Fibromyalgia (FMS) status, Adjusted for Demographic and Lifestyle factors, C8 Health Project, 2005-2006 (Continued)

\begin{tabular}{lllll}
\hline Former & $12,985(24.7 \%)$ & $284(25.2 \%)$ & $12,701(24.7 \%)$ & $1.06(0.91-1.23), .441$ \\
Exercise Program & & & & \\
$\quad$ No regularity (ref) & $36,113(68.7 \%)$ & $762(67.7 \%)$ & $35,351(68.8 \%)$ & - \\
$\quad$ Regular exercise & $16,422(31.3 \%)$ & $363(32.3 \%)$ & $16,059(31.2 \%)$ & - \\
Menopause occurred & $10,526(38.2 \%)$ & $631(61.0 \%)$ & $9895(37.3 \%)$ & $1.82(1.49,2.21),<.0001^{*}$ \\
\hline
\end{tabular}

Note: Column Percentages shown

* Significant at $p<0.05$

${ }^{a}$ Model adjusted for demographic covariates Age, Gender, Marital Status, Employment, Education level, White Race, and Household Income; Also adjusted for lifestyle covariates Tobacco Use and Current Alcohol consumption

demographic and lifestyle factors (AOR per unit BMI increase $=1.02$, CI 1.01, 1.03). Relative to those with a $\mathrm{BMI}<25$, participants who were obese were significantly more likely to have FMS $(\mathrm{AOR}=1.24, \mathrm{CI} 1.02,1.49$; and, $\mathrm{AOR}=1.43$, CI 1.18, 1.74, for BMI 30-35 and BMI $>35$, respectively). There was a $60 \%$ increase in FMS diagnosis for each additional comorbidity $(\mathrm{AOR}=1.60$, CI 1.54, 1.67 (Table 2); participants reporting a diagnosis

Table 2 Health Characteristics of a US adult population by Fibromyalgia (FMS) status, Adjusted for Demographic and Lifestyle factors, C8 Health Project, 2005-2006

\begin{tabular}{|c|c|c|c|c|}
\hline \multirow[t]{2}{*}{ Characteristic } & Total Population & FMS & Non-FMS & Adjusted Odds Ratio $^{a}$ \\
\hline & \multicolumn{3}{|l|}{ N (\%) / Mean (SD) } & OR $(\mathrm{Cl}), p$-value \\
\hline $\mathrm{BMI+}$ & $28.53(6.31)$ & $30.47(7.22)$ & $28.49(6.28)$ & $1.02(1.01-1.03), .001^{*}$ \\
\hline$\leq 24.99$ (ref) & $16,258(31.0 \%)$ & $279(24.8 \%)$ & 15,979 (31.1\%) & - \\
\hline $25.5-29.99$ & $18,200(34.6 \%)$ & $320(28.4 \%)$ & $17,880(34.8 \%)$ & $1.11(.932-1.31), .248$ \\
\hline $30-34.9$ & $10,704(20.4 \%)$ & $251(22.3 \%)$ & $10,453(20.3 \%)$ & $1.24(1.02-1.49), .028^{*}$ \\
\hline $35+$ & $7373(14.0 \%)$ & $275(24.4 \%)$ & $7098(13.8 \%)$ & $1.43(1.18-1.74), .0003^{*}$ \\
\hline Number of Comorbidities & $1.29(1.27)$ & $2.78(1.59)$ & $1.25(1.24)$ & $1.60(1.54-1.67),<.0001^{*}$ \\
\hline \multicolumn{5}{|l|}{ Comorbidity Index } \\
\hline None (ref) & 16,778 (31.9\%) & $58(5.2 \%)$ & $16,720(32.5 \%)$ & - \\
\hline 1 & 16,999 (32.4\%) & $177(15.7 \%)$ & $16,822(32.7 \%)$ & $2.38(1.77-3.21),<.0001^{*}$ \\
\hline 2 & $10,535(20.1 \%)$ & $301(26.8 \%)$ & $10,234(19.9 \%)$ & $5.16(3.88-6.86),<.0001^{*}$ \\
\hline $3+$ & $8223(15.7 \%)$ & $589(52.4 \%)$ & $7634(14.9 \%)$ & $10.46(7.90-13.84),<.0001$ \\
\hline Autoimmune disorder & $2192(4.2 \%)$ & $220(19.6 \%)$ & $1972(3.8 \%)$ & $3.43(2.89-4.06),<.0001^{*}$ \\
\hline Osteoarthritis & $4093(7.7 \%)$ & $411(35.5 \%)$ & $3782(7.1 \%)$ & $3.86(3.34-4.45),<.0001^{*}$ \\
\hline Allergies or Chronic Sinusitis & $24,470(46.6 \%)$ & $893(79.4 \%)$ & $23,577(45.9 \%)$ & $2.91(2.51-3.38),<.0001^{*}$ \\
\hline Kidney disorder & $5202(9.9 \%)$ & $226(20.1 \%)$ & 4976 (9.7\%) & $1.72(1.47-2.01),<.0001^{*}$ \\
\hline Respiratory disease & $7385(14.1 \%)$ & $342(30.4 \%)$ & 7043 (13.7\%) & $1.89(1.64-2.17),<.0001^{*}$ \\
\hline Cardiovascular disease & $4661(8.9 \%)$ & $148(13.2 \%)$ & $4513(8.9 \%)$ & $1.25(1.03-1.51), .027^{*}$ \\
\hline Liver disease & $684(1.3 \%)$ & $38(3.4 \%)$ & $646(1.3 \%)$ & $1.51(1.06-2.16), .022^{*}$ \\
\hline Endocrine Disorder & $4083(7.8 \%)$ & $264(23.5 \%)$ & 3819 (7.4\%) & $1.82(1.57-2.12),<.0001^{*}$ \\
\hline Diabetes & 4778 (9.1\%) & $157(14.0 \%)$ & $4621(9.0 \%)$ & $.978(.813-1.18), .810$ \\
\hline Frequent Headache & 10,105 (19.2\%) & 439 (39.0\%) & $9666(18.8 \%)$ & $2.03(1.78-2.31),<.0001^{*}$ \\
\hline Sleep Impairment Score ${ }^{b}$ & $4.00(3.56)$ & $7.32(3.29)$ & $3.93(3.53)$ & $1.23(1.21-1.25),<.0001^{*}$ \\
\hline Mood Disturbance Score ${ }^{m}$ & $4.59(3.70)$ & $7.25(3.40)$ & $4.53(3.68)$ & $1.19(1.17-1.21),<.0001^{*}$ \\
\hline
\end{tabular}

Note: Column Percentages shown

*Significant at $p<0.05$

a Model adjusted for demographic covariates Age, Gender, Marital Status, Employment, Education level, White Race, and Household Income; Also adjusted for lifestyle covariates Tobacco Use and Current Alcohol consumption

${ }^{b}$ Sleep impairment derived from responses to four individual questions regarding the frequency of short sleep, fitful sleep, insomnia and/or daytime somnolence; higher score indicates increased frequency of impairment

m Mood disturbance derived from responses to four individual questions regarding frequent mood swings, irritability, fatigue and/or inability to concentrate; higher score indicates increased frequency of disturbance 
of 3 or more comorbidities were 10.5 times more likely to have FMS (AOR $=10.46$, CI 7.90, 13.84) compared to those with no comorbidities. FMS was likewise strongly and positively associated with most chronic conditions evaluated, including in order of decreasing magnitude osteoarthritis (AOR $=3.86$, CI 3.34, 4.45), autoimmune disease $(\mathrm{AOR}=3.43, \mathrm{CI} 2.89,4.06)$, allergies/chronic sinusitis $(\mathrm{AOR}=2.91$, CI 2.51, 3.38), frequent headaches $(\mathrm{AOR}=2.03, \mathrm{CI} 1.78,2.31)$, and kidney $(\mathrm{AOR}=1.72, \mathrm{CI}$ $1.47,2.01)$, respiratory $(\mathrm{AOR}=1.89, \mathrm{CI} 1.64,2.17)$, endocrine $(\mathrm{AOR}=1.82$, $\mathrm{CI} 1.57,2.12)$, and liver $(\mathrm{AOR}=1.51, \mathrm{CI} 1.06,2.16)$ diseases.

FMS cases reported higher sleep $(M=7.3, \mathrm{SD}=3.3$ vs. $M=3.9, \mathrm{SD}=3.5)$ and mood disturbance scores $(M=7.3$, $\mathrm{SD}=3.4$ vs. $M=4.5, \mathrm{SD}=3.7$ ) than did controls (Table 2). After adjustment for demographic and lifestyle factors, FMS remained strongly and positively related to scores for both sleep ( $\mathrm{AOR}=1.23$, CI 1.21, 1.25), and mood disturbance $(\mathrm{AOR}=1.19$, CI 1.17, 1.21).

\section{Relation of CRP to FMS}

Mean CRP (mg/L) was significantly higher among FMS cases $(M=5.54, \mathrm{SD}=9.8)$ compared to controls
$(M=3.75, \mathrm{SD}=7.2)($ Table 3$) . \mathrm{CRP}$ serum level showed a positive association with FMS (unadjusted OR for highest vs. lowest quartile $=2.50$, CI 2.10, 2.97; P for trend <.0001); adjustment for demographic and lifestyle factors substantially attenuated but did not eliminate this association (AOR for highest vs. lowest quartile $=1.35$, CI 1.13, 1.62; P for trend <.0001). Analysis of CRP as a continuous variable yielded similar findings, with odds of FMS increasing by $2 \%$ for each one $\mathrm{mg} / \mathrm{L}$ of CRP rise (unadjusted $\mathrm{OR}=1.02$, CI 1.01, 1.02); adjustment for demographic and lifestyle factors slightly attenuated this association (AOR $=1.01$, CI 1.00, 1.01).

The addition of BMI and comorbidities to the model further weakened the relationship between CRP and FMS (AORs for highest vs. lowest CRP quartile $=1.17$ (CI 0.96, 1.42) and 1.10 (CI 0.92, 1.32), for BMI and comorbidities, respectively; and, combined OR $=1.07(\mathrm{CI}$ $0.88,1.30)$ ) suggesting that these factors may at least partially explain the observed associations (Table 3). The inclusion of mood disturbance and sleep impairment, separately and combined, only slightly attenuated the association of FMS to CRP after adjustment for demographic and lifestyle factors $(\mathrm{AOR}=1.01, \mathrm{CI} 1.00,1.01)$

Table 3 Model Statistics assessing the association between Fibromyalgia (FMS) and blood serum C-reactive Protein (CRP) quartile adjusting for BMI and Comorbidity index in a US adult population, 2005-2006 ( $N=52,535)$

\begin{tabular}{|c|c|c|c|c|c|c|c|c|}
\hline \multirow[t]{3}{*}{ Characteristic } & \multirow[t]{2}{*}{ Total } & \multirow[t]{2}{*}{ FMS } & \multirow{3}{*}{$\begin{array}{l}\text { Non- } \\
\text { FMS }\end{array}$} & \multicolumn{5}{|l|}{ Models } \\
\hline & & & & \multicolumn{5}{|c|}{ Odds Ratio (Cl) ( $p$-value) } \\
\hline & \multicolumn{2}{|c|}{ N (\%) / Mean (SD) } & & Crude $^{e}$ & $\begin{array}{l}\text { Adjusted for } \\
\text { Demographic } \\
\text { and Lifestyle } \\
\text { Factors (Model 1) }\end{array}$ & $\begin{array}{l}\text { Model } \\
1 \pm \mathrm{BMI}^{\mathrm{g}}\end{array}$ & $\begin{array}{l}\text { Model } 1 \pm \\
\text { comorbidities }^{h}\end{array}$ & $\begin{array}{l}\text { Model } 1 \pm \text { BMI } \\
\& \text { comorbidities }\end{array}$ \\
\hline CRP level (mg/L) & $3.79(7.22)$ & $5.54(9.77)$ & $\begin{array}{l}3.75 \\
(7.15)\end{array}$ & $\begin{array}{l}1.02(1.01-1.02) \\
\left(<.0001^{*}\right)\end{array}$ & $\begin{array}{l}1.01(1.00-1.01) \\
\left(.004^{*}\right)\end{array}$ & $\begin{array}{l}1.01(1.00-1.01) \\
(.059)\end{array}$ & $\begin{array}{l}1.00(.997-1.01) \\
(.267)\end{array}$ & $\begin{array}{l}1.00(.997-1.01) \\
(.279)\end{array}$ \\
\hline $\begin{array}{l}\text { CRP Quartile } 1^{a} \\
\text { (ref) }\end{array}$ & $14,516(27.6 \%)$ & 192 (17.1\%) & $\begin{array}{l}14,324 \\
(27.9 \%)\end{array}$ & - & - & - & - & - \\
\hline Quartile $2^{b}$ & $12,221(23.3 \%)$ & $214(19.0 \%)$ & $\begin{array}{l}12,007 \\
(23.4 \%)\end{array}$ & $\begin{array}{l}1.33(1.09-1.62) \\
\left(.004^{*}\right)\end{array}$ & $\begin{array}{l}1.13(0.92-1.38) \\
(.243)\end{array}$ & $\begin{array}{l}1.07(0.88-1.32) \\
(.473)\end{array}$ & $\begin{array}{l}1.04(0.85-1.28) \\
(.686)\end{array}$ & $\begin{array}{l}1.03(0.84-1.27) \\
(.750)\end{array}$ \\
\hline Quartile $3^{c}$ & $12,755(24.3 \%)$ & 297 (26.4\%) & $\begin{array}{l}12,458 \\
(24.2 \%)\end{array}$ & $\begin{array}{l}1.78(1.48-2.14) \\
\left(<.0001^{*}\right)\end{array}$ & $\begin{array}{l}1.23(1.02-1.48) \\
\left(.032^{*}\right)\end{array}$ & $\begin{array}{l}1.12(0.92-1.37) \\
(.244)\end{array}$ & $\begin{array}{l}1.08(0.89-1.30) \\
(.452)\end{array}$ & $\begin{array}{l}1.06(0.87-1.29) \\
(.576)\end{array}$ \\
\hline Quartile $4^{d}$ & $13,043(24.8 \%)$ & $422(37.5 \%)$ & $\begin{array}{l}12,621 \\
(24.6 \%)\end{array}$ & $\begin{array}{l}2.50(2.10-2.97) \\
\left(<.0001^{*}\right)\end{array}$ & $\begin{array}{l}1.35(1.13-1.62) \\
\left(.0009^{*}\right)\end{array}$ & $\begin{array}{l}1.17(0.96-1.42) \\
(.120)\end{array}$ & $\begin{array}{l}1.10(0.92-1.32) \\
(.310)\end{array}$ & $\begin{array}{l}1.07(0.88-1.30) \\
(.510)\end{array}$ \\
\hline $\begin{array}{l}\text { Wald } X^{2}, p \text { for } \\
\text { trend }^{t}\end{array}$ & & & & $118.42,\left(<.0001^{*}\right)$ & $17.48,\left(<.0001^{*}\right)$ & $4.02,\left(.045^{*}\right)$ & $3.44,(.064)$ & $1.23,(.269)$ \\
\hline
\end{tabular}

*Significant at $p<0.05$

${ }^{\mathrm{a}} \mathrm{C}$-reactive protein. $15-.80 \mathrm{mg} / \mathrm{L}$

${ }^{\mathrm{b}} \mathrm{C}$-reactive protein.81-1.80 mg/L

${ }^{\mathrm{C}} \mathrm{C}$-reactive protein $1.81-4.20 \mathrm{mg} / \mathrm{L}$

${ }^{\mathrm{d}} \mathrm{C}$-reactive protein $4.21-250.6 \mathrm{mg} / \mathrm{L}$

thinear trend

e $\left(R^{2=}=.003\right)$

${ }^{f}$ Full Model adjusted for demographic covariates Age, Gender, Marital Status, Employment, Education level, White Race, and Household Income; Also adjusted for lifestyle covariates Tobacco Use and Current Alcohol consumption $\left(\mathrm{R}^{2}=.031\right)$

${ }^{\mathrm{g}}\left(\mathrm{R}^{2}=.031\right)$

hModel adjusted for Comorbidity index; index included diabetes, other endocrine disorders (thyroid, Addison's, and Cushing's disease), kidney disease, respiratory conditions (asthma, emphysema, chronic obstructive pulmonary disorder, bronchitis), osteoarthritis, heart disease, liver disease, autoimmune disorders (defined as having any diagnoses of immune disease, lupus, or rheumatoid arthritis), sleep apnea, irritable bowel syndrome, allergies or frequent sinusitis, or frequent recurrent headache $\left(R^{2}=.041\right)$

${ }^{r}\left(R^{2}=.041\right)$ 
Table 4 Model Statistics assessing the association between Fibromyalgia (FMS) and blood serum C-reactive Protein (CRP) quartile adjusting for Sleep impairment and Mood disturbance in a US adult population, 2005-2006 ( $N=52,535)$

\begin{tabular}{|c|c|c|c|c|c|}
\hline & \multicolumn{5}{|l|}{ Models } \\
\hline & \multicolumn{5}{|l|}{ Odds Ratio (Cl) ( $p$-value) } \\
\hline & Crude $^{e}$ & $\begin{array}{l}\text { Adjusted for Demographic and } \\
\text { Lifestyle Factors (Model 1) }\end{array}$ & Model $1 \pm$ Mood $^{g}$ & Model $1 \pm$ Sleep $^{h}$ & $\begin{array}{l}\text { Model } 1+\text { Mood } \\
\text { \& Sleep }\end{array}$ \\
\hline CRP level (mg/L) & $1.02(1.01-1.02)\left(<.0001^{*}\right)$ & $1.01(1.00-1.01)\left(.004^{*}\right)$ & $\begin{array}{l}1.01(1.00-1.01) \\
\left(.021^{*}\right)\end{array}$ & $\begin{array}{l}1.01(1.00-1.01) \\
\left(.035^{*}\right)\end{array}$ & $\begin{array}{l}1.01(1.00-1.01) \\
\left(.031^{*}\right)\end{array}$ \\
\hline CRP Quartile $1^{\mathrm{a}}$ (ref) & - & - & - & - & - \\
\hline Quartile $2^{\mathrm{b}}$ & $1.33(1.09-1.62)\left(.004^{*}\right)$ & $1.13(0.92-1.38)(.243)$ & $1.12(0.91-1.36)(.287)$ & $1.10(0.90-1.35)(.357)$ & $1.10(0.90-1.34)(.363)$ \\
\hline Quartile $3^{c}$ & $1.78(1.48-2.14)\left(<.0001^{*}\right)$ & $1.23(1.02-1.48)\left(.032^{*}\right)$ & $1.20(1.00-1.45)(.056)$ & $1.18(0.98-1.43)(.083)$ & $1.18(0.97-1.42)(.093)$ \\
\hline Quartile $4^{d}$ & $2.50(2.10-2.97)\left(<.0001^{*}\right)$ & $1.35(1.13-1.62)\left(.0009^{*}\right)$ & $\begin{array}{l}1.28(1.07-1.53) \\
\left(.007^{*}\right)\end{array}$ & $\begin{array}{l}1.26(1.05-1.51) \\
\left(.011^{*}\right)\end{array}$ & $\begin{array}{l}1.24(1.03-1.48) \\
\left(.021^{*}\right)\end{array}$ \\
\hline $\begin{array}{l}\text { Wald } X^{2}, p \text { for } \\
\text { trend }^{t}\end{array}$ & $118.42,\left(<.0001^{*}\right)$ & $17.48,\left(<.0001^{*}\right)$ & $11.06,\left(.0009^{*}\right)$ & $10.40,\left(.001^{*}\right)$ & $8.25,\left(.004^{*}\right)$ \\
\hline
\end{tabular}

*Significant at $p<0.05$

${ }^{\mathrm{a} C} \mathrm{C}$-reactive protein $.15-.80 \mathrm{mg} / \mathrm{L}$

${ }^{\mathrm{b}} \mathrm{C}$-reactive protein $.81-1.80 \mathrm{mg} / \mathrm{L}$

${ }^{\mathrm{C}} \mathrm{C}$-reactive protein $1.81-4.20 \mathrm{mg} / \mathrm{L}$

${ }^{\mathrm{d}} \mathrm{C}$-reactive protein $4.21-250.6 \mathrm{mg} / \mathrm{L}$

thinear trend

${ }^{\mathrm{e}}\left(\mathrm{R}^{2=}=.003\right)$

fFull Model adjusted for demographic covariates Age, Gender, Marital Status, Employment, Education level, White Race, and Household Income; Also adjusted for lifestyle covariates Tobacco Use and Current Alcohol consumption $\left(R^{2}=.031\right)$

${ }^{9}$ Mood disturbance derived from responses to four individual questions regarding frequent mood swings, irritability, fatigue and/or inability to concentrate $\left(\mathrm{R}^{2}=.035\right)$

${ }^{\mathrm{h}}$ Sleep impairment derived from responses to four individual questions regarding the frequency of short sleep, fitful sleep, insomnia and/or daytime somnolence $\left(R^{2}=.037\right)$

${ }^{r}\left(R^{2}=.038\right)$

(Table 4). These findings suggest that any mediating effect of these factors was modest.

Additional adjustment for statin use, PFOA, and female reproductive characteristics (menopausal status, age at menarche, and parity) did not appreciably change the relationship between CRP and FMS. Similarly, exclusion of those with rheumatoid arthritis $(n=1821)$ and all autoimmune conditions $(n=2192)$ did not appreciably affect risk estimates. Likewise, we found no evidence for a modifying effect of age, tobacco use, gender, obesity, menopausal status, or other factors on the relationship between CRP and FMS.

\section{Discussion}

This is the first large, population-based investigation to examine the relationship between CRP and FMS, to assess the potential influence of BMI and comorbid conditions on this relationship, and to evaluate the potential mediating role of mood and sleep impairment. In this cross-sectional study of a large population in the U.S., CRP serum level showed a positive association with FMS, which remained significant after adjustment for multiple demographic and lifestyle factors, including age, gender, education level, employment, marital status, alcohol, and tobacco use. Adjustment for BMI and comorbid conditions substantially attenuated this relationship. These findings are broadly consistent with those from a recent cross-sectional investigation of 5110 Norwegian men; cases with FMS/Chronic Fatigue Syndrome
(CFS) showed a strong, positive relationship to high sensitivity-CRP level (FMS/CFS $M=4.79 \mathrm{mg} / \mathrm{L}$; OR for $\geq 10 \mathrm{mg} / \mathrm{L}$ vs. $<1 \mathrm{mg} / \mathrm{L}=2.6,95 \% \mathrm{CI}: 1.4,4.6, p=.002$; P for trend $=.006$ ) after adjustment for age, education, smoking, and cholesterol medication [27]. In contrast, as stated in the introduction, findings from smaller casecontrol studies examining the association between CRP and FMS have been inconsistent [24, 26, 28, 30], perhaps due to varying sample sizes and differing selection criteria among controls; of the four case-control studies published to date, only one reported significantly higher CRP levels in FMS patients compared to healthy controls after adjustment for age, sex, and race [24]. Mean CRP values found among those with FMS $(M=5.54 \mathrm{mg} / \mathrm{L})$ nor non-FMS controls $(M=3.75 \mathrm{mg} / \mathrm{L})$ in our study are nearly within the range for normal CRP values $(0.0$ $5.0 \mathrm{mg} / \mathrm{L}$ ) [28] and also within ranges of those found in most previous studies (mean FMS high-sensitivity CRP range $=2.6-10.6 \mathrm{mg} / \mathrm{L}$; mean FMS CRP range $=1.0$ $4.7 \mathrm{mg} / \mathrm{L})[24,26,28,30]$. One exception, however, was that reported by Rus et al. $(1.0 \pm 0.75)$ in a Spanish population of women with FMS [28]; in addition to differences in CRP by BMI status, other possible explanations for the comparative value found in our study may include gender variations and/or the influence of FMS comorbidities.

As indicated above, the inclusion of BMI in our model substantially attenuated the association of FMS to CRP, suggesting BMI may have largely explained the elevated 
CRP levels in FMS; this was second to the inclusion of comorbidities. Obesity is a major determinant of elevated CRP in multiple populations [50,51]. Two studies to date have considered the potential contribution of BMI to the profile of CRP in FMS, with one demonstrating a strong and positive correlation [24] while another demonstrated overweight women with FMS had a higher mean CRP compared to normal weight women with FMS $(N=25 ; \quad \mathrm{M}=3.1 \pm 1.4 \mathrm{mg} / \mathrm{L}$ and $\mathrm{M}=1.0 \pm 0.8 \mathrm{mg} / \mathrm{L}$, respectively). Additionally, in agreement with our study, others have suggested a mediating effect of BMI on the relation between CRP and FMS; FMS symptom improvement has followed weight loss among several FMS cases [52], and a longitudinal study revealed regular exercise and maintenance of body weight lowered the risk of FMS [53]. Additionally, one case-control study found that after adjustment for demographic factors, BMI was the only significant contributor in a model exploring the relation between CRP and FMS $(r=.062, p<0.001)$ [24].

Ours is the first study to assess the contribution of multiple comorbidities on the relation of CRP and FMS. Comorbidities characterized by pain may contribute to the development of FMS; shared pain mechanisms between FMS and other conditions with a similar underlying pathophysiology (e.g., tension headache) or as a comorbidity characterized by inflammation or ongoing peripheral damage (e.g., autoimmune disorders and osteoarthritis) and FMS have recently been explored $[4,39,54]$. Our study found that as the number of comorbidities increased, the odds of FMS increased as well.

Additionally, the presence of sleep impairment and mood disturbance modestly attenuated, but did not eliminate, the association between CRP and FMS. No existing studies have examined the role of sleep impairment on this relationship. We found no evidence supporting mood disorder as an effect modifier, similar to a study finding where CRP level did not differ by psychiatric status among those with FMS [26]. The influence of sleep impairment and mood disturbance on the relation between CRP and FMS in our study likely reflects a bidirectional relationship.

The prevalence rate of FMS in our study (2.1\%) mirrored recent estimates for the U.S. population [3, 55]. Consistent with previous studies, we found the likelihood of FMS diagnosis was increased in middle-aged females $[3,5]$. However, in contrast to findings from two large studies $[3,56]$, those who were married or reported higher levels of education were more likely to report FMS. Females who had experienced menopause were 1.8 times more likely to have FMS than those who had not; however, a lack of epidemiological research exists in the topical area of sex hormones, neurotransmitters, and FMS. We observed no significant association between age at menarche or parity and FMS, unlike a German case-control study of 653 middle-aged women (FMS cases $=36$ ) which found, after controlling for age, those with FMS had significantly later menarche and were less likely to have ever been pregnant [57].

In agreement with previous cross-sectional and longitudinal studies $[3,53,58]$, BMI was strongly and positively related to reported FMS in this large U.S. population. Additionally, higher BMI has been associated with an increased risk of FMS after adjustment for mood and/or other health factors [3, 53], including familial FMS diagnosis [58]. Likewise, consistent with findings from a recent cross-sectional study of a nationally representative sample of U.S. adults $(N=8446$, FMS cases $=201)$ [3] FMS showed significant positive associations to multiple comorbid conditions in our study after adjustment for demographic and lifestyle factors; these included cardiovascular disease, rheumatoid arthritis and other autoimmune disorders, kidney, respiratory and liver diseases, and frequent headache. Osteoarthritis was also associated with a nearly 4-fold likelihood of FMS, dissimilar from a large, national analysis of U.S. electronic medical records $(N=587,961$, FMS cases $=4296)$ of only a (unadjusted) 2-fold likelihood [59]. In contrast to other large studies of U.S. adults [3, 59], diabetes was not associated with FMS in this sample of adults.

The strengths of this study were its high response rate, population-based design, and large sample size; this was the largest comprehensive community study conducted to date in the Appalachian region of the U.S. We were able to control for a large number of potential confounders, including comorbid conditions. Misclassification of CRP was unlikely due to standardized assay procedures used.

This study targeted a population of predominantly white Appalachian adults in the U.S., potentially limiting generalizability. Possible misclassification of FMS may have occurred depending on time the participant was diagnosed and the physician's awareness and use of the American College of Rheumatology classification criteria for FMS diagnosis, first established in 1990. In particular, poor or incomplete recognition of FMS by healthcare providers may have led to under-ascertainment [60]. However, such under-ascertainment would be expected to bias the observed associations toward the null, and thus would indicate that the magnitudes of relationships in this study are possibly stronger than those which we reported. FMS, in addition to most other assessed health conditions in this cross-sectional survey, was based on self-report without medical chart review, possibly leading to response or misclassification bias. To our knowledge, no clinical validation study has assessed the agreement between self-report and medical recordverified data to understand how patients accept and report their FMS diagnosis, especially in the presence of targeted 
interventions. Additionally, our assessment of the potential relationship between CRP and FMS could be strengthened by inclusion of symptom onset and response to interventions, for which data was not available.

Unmeasured confounding may have also contributed to our findings, although our ability to control for a large number of both known and potential risk factors for FMS diminishes this possibility. Our study also lacked specific information on certain conditions previously linked to FMS, including sleep apnea and irritable bowel syndrome. Although contact with former residents of counties used for study inclusion was attempted, some may not have participated in the study, possibly introducing sampling bias. Lastly, we cannot draw any conclusions regarding causality due to the lack of a temporal component in our cross-sectional study design.

In this large cross-sectional study, we observed a significant, positive association between serum CRP and diagnosed FMS, which was largely explained by elevated BMI and chronic comorbid conditions. Adjustment for sleep and mood disturbance only modestly attenuated this association, suggesting that BMI and chronic comorbid conditions may largely account for elevated CRP levels among FMS patients over the presence of sleep and mood disturbance. While the clinical utility of CRP for FMS diagnosis remains elusive at best, the current work contributes to existing literature by more appropriately assigning responsibility of inflammatory processes often present in those with FMS to co-occurring chronic conditions and other factors associated with chronic disease. Further prospective research is needed to determine the relation of CRP and other inflammatory markers to the development and progression of FMS in the presence of the potentially complex roles of obesity and comorbidities.

\section{Conclusion}

Findings from this large cross-sectional study indicate the significant positive cross-sectional association of Fibromyalgia to serum CRP may be explained, in part, by factors such as BMI, comorbidity, impaired mood, and sleep disturbance. Prospective research is needed to confirm this, and better clarify the potential mediating influences on the relationship between Fibromyalgia and CRP.

\section{Abbreviations}

BMI: Body Mass Index; CRP: C-reactive protein; FMS: Fibromyalgia syndrome; PFOA: Perfluorooctanoic Acid; WVU: West Virginia University

\section{Funding}

This research was fulfilled with funding source National Institutes of Health National Institute of General Medical Sciences grant T32 GM081741.

\section{Availability of data and materials}

The data that support the findings of this study are available from West Virginia University but restrictions apply to the availability of these data, which were used under license for the current study, and so are not publicly available. Data are however available from the authors upon reasonable request and with permission of West Virginia University.

\section{Authors' contributions}

TF conducted data analysis and wrote the manuscript with $\mathrm{Kl}$; US and $\mathrm{CL}$ provided additional input on manuscript and statistical analysis. All authors have read and approved of the final manuscript.

\section{Ethics approval and consent to participate}

Consent to participate was given by Brookmar, Inc. (Parkersburg, WV) and the Wood County, WV, Circuit Court (http://www.c8sciencepanel.org/ index.html). The West Virginia University Institutional Review Board approved the use of de-identified data by investigators, and consents use of de-identified data for publication.

\section{Consent for publication}

Not Applicable.

\section{Competing interests}

The authors declare no competing interests.

\section{Publisher's Note}

Springer Nature remains neutral with regard to jurisdictional claims in published maps and institutional affiliations.

\section{Author details}

'Department of Family and Community Medicine, Center for Integrative Medicine, University of Maryland School of Medicine, 520 W. Lombard St. East Hall, Baltimore, MD 21201-1603, USA. ²Department of Epidemiology, West Virginia University School of Public Health, P.O. Box 9190, Morgantown, W 26506-9190, USA. ${ }^{3}$ Department of Pharmaceutical Systems and Policy, West Virginia University School of Pharmacy, P.O. Box 9500, Morgantown, WV 26506-9500, USA. Department of Biostatistics, West Virginia University School of Public Health, P.O. Box 9190, Morgantown, WV 26506-9190, USA.

${ }^{5}$ Center for the Study of Complementary and Alternative Therapies, University of Virginia Health System, P.O. Box 800782, McLeod Hall, Charlottesville, VA 22908-0782, USA.

Received: 10 August 2016 Accepted: 27 June 2017

Published online: 07 July 2017

\section{References}

1. Manchikanti L, Singh V, Datta S, Cohen SP, Hirsch JA. American Society of Interventional Pain Physicians. Comprehensive review of epidemiology, scope, and impact of spinal pain. Pain Physician. 2009;12:E35-70.

2. White KP, Harth M. Classification, epidemiology, and natural history of fibromyalgia. Curr Pain Headache Rep. 2001;5(4):320-9. doi:10.1007/s11916001-0021-2

3. Walitt B, Nahin RL, Katz RS, Bergman MJ, Wolfe F. The prevalence and characteristics of fibromyalgia in the 2012 National Health Interview Survey. PLoS One. 2015;10(9):e0138024. doi:10.1371/journal.pone.0138024. PubMed PMID: 26379048

4. Clauw DJ. Fibromyalgia and related conditions. Mayo Clin Proc. 2015;90(5): 680-92. doi:10.1016/j.mayocp.2015.03.014. PubMed PMID: 25939940

5. NIH. Questions and answers about fibromyalgia 2014 [cited 2014 July]. Available from: http://www.niams.nih.gov/Health_Info/Fibromyalgia/default.asp.

6. Bennett RM, Jones J, Turk DC, Russell IJ, Matallana L. An internet survey of 2,596 people with fibromyalgia. BMC Musculoskelet Disord. 2007;8:27. doi: 10.1186/1471-2474-8-27. PubMed PMID: 17349056; PubMed Central PMCID: PMCPMC1829161

7. Arnold LM, Hudson J, Hess EV, Ware AE, Fritz DA, Auchenbach MB, et al. Family study of fibromyalgia. Arthritis Rheum. 2004;50(3):944-52. doi:10. 1002/art.20042. Epub 2004/03/17. PubMed PMID: 15022338

8. Metyas SK, Solyman JS, Arkfeld DG. Inflammatory fibromyalgia: is it real? Curr Rheumatol rev. 2015. PubMed PMID: 26002453.

9. Bjersing $\mathrm{L}$, Bokarewa MI, Mannerkorpi K. Profile of circulating microRNAs in fibromyalgia and their relation to symptom severity: an exploratory study. Rheumatol Int. 2015:35(4):635-42. doi:10.1007/s00296-014-3139-3. PubMed PMID: 25261961 
10. Fok E, Sandeman SR, Guildford AL, Martin YH. The use of an IL-1 receptor antagonist peptide to control inflammation in the treatment of corneal Limbal epithelial stem cell deficiency. Biomed Res Int. 2015;2015:516318. doi:10.1155/2015/516318. PubMed PMID: 25705668; PubMed Central PMCID: PMC4330955

11. Pepys MB, Hirschfield GM. C-reactive protein: a critical update. J Clin Invest. 2003;111(12):1805-12. doi:10.1172/JCl18921. PubMed PMID: 12813013; PubMed Central PMCID: PMC161431

12. Bucova M, Bernadic M, Buckingham T. C-reactive protein, cytokines and inflammation in cardiovascular diseases. Bratislavske lekarske listy. 2008; 109(8):333-40. PubMed PMID: 18837239

13. Zhang J-M, An J. Cytokines, inflammation and pain. Int Anesthesiol Clin. 2007 45(2):27-37. doi:10.1097/AIA.0b013e318034194e. PubMed PMID: PMC2785020

14. FibroCenter. Fibromyalgia pain is different: Pfizer; 2015 [cited 2015]. Available from: http://www.fibrocenter.com/fibromyalgia-pain.aspx.

15. Maes M, Libbrecht I, Van Hunsel F, et al. The immune-inflammatory pathophysiology of fibromyalgia: increased serum soluble gp130, the common signal transducer protein of various neurotropic cytokines. Psychoneuroendocrinology. 1999;24(4):371-83.

16. Wallace DJL-IM, Hallegua D, Silverman S, Silver MH, Weisman MH. Cytokines play an aetiopathogenetic role in fibromyalgia: a hypothesis and pilot study. Rheumatology (Oxford). 2001;40(7):743-9.

17. Gur AKM, Erdogan S, Nas K, Cevik R, Sarac AJ. Regional cerebral flow and cytokines in young females with fibromyalgia. Clinical and experimental rheumatology JID - 8308521. 2002;20(6):753-60.

18. Gur AKM, Nas K, Remzi C, Denli A, Sarac J. Cytokines and depression in cases with fibromyalgia. J Rheumatol. 2002;29(2):358-3561.

19. Hein GFS. Are advanced glycaton end-product-modified proteins of pathogentic importance in fibromyalgia. Rheumatology. 2002:41(10):1163-7.

20. Pache MOJ, Genth E, Mierau R, Kube T, Flammer J. Increased plasma endothilin-1 levels in fibromyalgia syndrome. Rheumatology. 2003:42:493-4.

21. Bagis $S$, et al. Free radicals and antioxidants in primary fibromyalgia: an oxidative stress disorder? Rheumatol Int. 2005;25:188-90.

22. Salemi SRJ, Wollina U, et al. Detection of interleukin 1 beta (IL-beta), IL0-6, and tumor necrosis factor-alpha in skin of patients with fibromyalgia. J Rheumatol. 2003;30(1):146-50.

23. Kadetoff DF, Lampa JF, Westman MF, Andersson MF, Kosek E. Evidence of central inflammation in fibromyalgia-increased cerebrospinal fluid interleukin-8 levels. 0328

24. Xiao YHW, Michalek JE, Russell IJ. Elevated serum high-sensitivity C-reactive protein levels in fibromyalgia syndrome patients correlate with body mass index, interleukin-6, interleukin-8, erythrocyte sedimentation rate. 2013.

25. Kaufmann IF, Schelling GF, Eisner CF, Fau RHP, Beyer AF, Krauseneck TF, et al. Decrease in adhesion molecules on polymorphonuclear leukocytes of patients with fibromyalgia. 0924.

26. Bazzichi LRA, Massimetti $G$, et al. Cytokine patterns in fibromyalgia and their correlation with clinical manifestations. Clin and Experim Rheum. 2007;25(2): 225-30.

27. Lund Haheim L, Nafstad P, Olsen I, Schwarze P R, KS. C-reactive protein variations for different chronic somatic disorders. 1001

28. Rus A, Molina F, Gasso M, Camacho MV, Peinado MA, Moral ML. Nitric Oxide, Inflammation, Lipid Profile, and Cortisol in Normal- and Overweight Women With Fibromyalgia. Biol Res Nurs. 2015. doi: 10.1177/1099800415591035. PubMed PMID: 26134428.

29. Amel Kashipaz M, Swinden D, Todd I, Powell R. Normal production of inflammatory cytokines in chronic fatigue and fibromyalgia syndromes determined by intracellular cytokine staining in short-term cultured blood mononuclear cells. Clin Exp Immunol. 2003;132(2):360-5. PubMed PMID: 12699429; PubMed Central PMCID: PMCPMC1808704

30. Afsar B, Burucu R. Urinary albumin, protein excretion and circadian blood pressure in patients with fibromyalgia. Rheumatol Int. 2013;33:2391-8. doi: 10.1007/s00296-013-2748-6.

31. Irwin MR, Olmstead R, Carroll JE. Sleep disturbance, sleep duration, and inflammation: a systematic review and meta-analysis of cohort studies and experimental sleep deprivation. Biol Psychiatry. 2015. doi: 10.1016/j.biopsych. 2015.05.014. PubMed PMID: 26140821

32. Consoli G, Marazziti D, Ciapparelli A, Bazzichi L, Massimetti G, Giacomelli C, et al. The impact of mood, anxiety, and sleep disorders on fibromyalgia. Compr Psychiatry. 2012;53(7):962-7. http://dx.doi.org/10.1016/j.comppsych.2012.03.008

33. Okifuji A, Donaldson GW, Barck L, Fine PG. Relationship between fibromyalgia and obesity in pain, function, mood, and sleep. J Pain. 2010;
11(12):1329-37. doi:10.1016/j.jpain.2010.03.006. PubMed PMID: 20542742; PubMed Central PMCID: PMCPMC2939916

34. Dell'Osso L, Bazzichi L, Baroni S, Falaschi V, Conversano C, Carmassi C, et al. The inflammatory hypothesis of mood spectrum broadened to fibromyalgia and chronic fatigue syndrome. Clin Exp Rheumatol. 2015;33 Suppl 88(1): 109-16. PubMed PMID: 25786052

35. Okifuji A, Bradshaw DH, Olson C. Evaluating obesity in fibromyalgia: neuroendocrine biomarkers, symptoms, and functions. Clin Rheumatol. 2009;28(4):475-8. doi:10.1007/s10067-009-1094-2. PubMed PMID: 19172342; PubMed Central PMCID: PMCPMC2668698

36. Frisbee SJ, Brooks AP Jr, Maher A, Flensborg P, Arnold S, Fletcher T, et al. The $C 8$ health project: design, methods, and participants. Environ Health Perspect. 2009;117(12):1873-82. doi:10.1289/ehp.0800379. Epub 2010/01/06. PubMed PMID: 20049206; PubMed Central PMCID: PMCPMC2799461

37. Ursini F, Naty S, Grembiale R. Fibromyalgia and obesity: the hidden link. Rheumatol Int. 2011;31(11):1403-8. doi:10.1007/s00296-011-1885-z.

38. Giacomelli C, Talarico R, Bombardieri S, Bazzichi $L$. The interaction between autoimmune diseases and fibromyalgia: risk, disease course and management. Expert Rev Clin Immunol. 2013;9(11):1069-76. doi:10.1586/1744666X.2013. 849440. PubMed PMID: 24168413

39. Staud R. Evidence for shared pain mechanisms in osteoarthritis, low back pain, and fibromyalgia. Curr Rheumatol Rep. 2011;13(6):513-20. doi:10.1007/ s11926-011-0206-6. PubMed PMID: 21833699

40. Wolfe F, Michaud K, Li T, Katz RS. Chronic conditions and health problems in rheumatic diseases: comparisons with rheumatoid arthritis, noninflammatory rheumatic disorders, systemic lupus erythematosus, and fibromyalgia. J Rheumatol. 2010;37(2):305-15. doi:10.3899/jrheum.090781. PubMed PMID: 20080915

41. Ozgocmen S, Cimen OB, Ardicoglu O. Relationship between chest expansion and respiratory muscle strength in patients with primary fibromyalgia. Clin Rheumatol. 2002;21(1):19-22. PubMed PMID: 11954878

42. Yang TY, Chen CS, Lin CL, Lin WM, Kuo CN, Kao CH. Risk for irritable bowel syndrome in fibromyalgia patients: a national database study. Medicine (Baltimore). 2015;94(10):e616. doi:10.1097/MD.0000000000000616. PubMed PMID: 25761187

43. Yanmaz MN, Mert M, Korkmaz M. The prevalence of fibromyalgia syndrome in a group of patients with diabetes mellitus. Rheumatol Int. 2012;32(4):8714. doi:10.1007/s00296-010-1618-8. PubMed PMID: 21221595

44. Michaud K, Wolfe F. The association of rheumatoid arthritis and its treatment with sinus disease. J Rheumatol 2006; 33(12):2412-2415. PubMed PMID: 17143978

45. Tietjen GE, Brandes JL, Peterlin BL, Eloff A, Dafer RM, Stein MR, et al. Allodynia in migraine: association with comorbid pain conditions. Headache. 2009;49(9): 1333-44. doi:10.1111/j.1526-4610.2009.01521.x. PubMed PMID: 19788473

46. Martinez-Jauand M, Sitges C, Femenia J, Cifre I, Gonzalez S, Chialvo D, et al. Age-of-onset of menopause is associated with enhanced painful and nonpainful sensitivity in fibromyalgia. Clin Rheumatol. 2013;32(7):975-81. doi:10. 1007/s10067-013-2212-8. PubMed PMID: 23417348

47. Goesling J, Brummett CM, Meraj TS, Moser SE, Hassett AL, Ditre JW. Associations between pain, current tobacco smoking, depression, and fibromyalgia status among treatment-seeking chronic pain patients. Pain Med. 2015;16(7):1433-42. doi:10.1111/pme.12747. PubMed PMID: 25801019

48. Prasad K. C-reactive protein (CRP)-lowering agents. Cardiovascular drug reviews. 2006;24(1):33-50. doi:10.1111/j.1527-3466.2006.00033.x. Epub 2006/ 08/31. PubMed PMID: 16939632

49. $\mathrm{NIH}$. Classification of Overweight and Obesity by BMI, Waist Circumference, and Associated Disease Risks: National Heart, Lung, and Blood Institute [cited 2015 February 7]. Available from: https://www.nhlbi.nih.gov/health/ educational/lose_wt/BMl/bmi_dis.htm.

50. Aronson D, Bartha P, Zinder O, Kerner A, Markiewicz W, Avizohar O, Brook GJ, Levy Y. Obesity is the major determinant of elevated C-reactive protein in subjects with the metabolic syndrome. Int J Obes Relat Metab Disord. 2004;28(5):674-9.

51. Kao TW, Lu IS, Liao KC, Lai HY, Loh CH, Kuo HK. Associations between body mass index and serum levels of C-reactive protein. S Afr Med J. 2009;99(5):326-30.

52. Deodhar AA, Fisher RA, Blacker CV, Woolf AD. Fluid retention syndrome and fibromyalgia. Br J Rheumatol. 1994;33(6):576-82. PubMed PMID: 8205408

53. Mork PJ, Vasseljen O, Nilsen TI. Association between physical exercise, body mass index, and risk of fibromyalgia: longitudinal data from the Norwegian Nord-Trondelag health study. Arthritis Care Res (Hoboken). 2010;62(5):611-7. doi:10.1002/acr.20118. PubMed PMID: 20191480 
54. Sanchez del Rio-Gonzalez M. Chronic migraine: pathophysiology. Rev Neurol. 2012;54 Suppl 2:S13-9. PubMed PMID: 22532238

55. Lawrence R, Felson D, Helmick CG, Arnold LM, Choi H, Deyo RA, et al. Estimates of the prevalence of arthritis and other rheumatic conditions in the United States. Part II. (0004-3591 (Print)). doi: D-NLM: NIHMS347693 D NLM: PMC3266664 EDAT- 2008/01/01 09:00 MHDA- 2008/03/11 09:00 CRDT2008/01/01 09:00 AID - 10.1002/art.23176 [doi] PST - ppublish.

56. White KP, Speechley M, Harth M, Ostbye T. The London fibromyalgia epidemiology study: the prevalence of fibromyalgia syndrome in London. Ontario J Rheumatol. 1999;26(7):1570-6. Epub 1999/07/16. PubMed PMID: 10405947

57. Schochat T, Beckmann C. Sociodemographic characteristics, risk factors and reproductive history in subjects with fibromyalgia-results of a populationbased case-control study. Z Rheumatol. 2003;62(1):46-59. doi:10.1007/ s00393-003-0447-5. PubMed PMID: 12624804

58. Wright LJ, Schur E, Noonan C, Ahumada S, Buchwald D, Afari N. Chronic pain, overweight, and obesity: findings from a community-based twin registry. J Pain. 2010;11(7):628-35. doi:10.1016/j.jpain.2009.10.004. PubMed PMID: 20338816; PubMed Central PMCID: PMCPMC2892725

59. Masters ET, Mardekian J, Emir B, Clair A, Kuhn M, Silverman SL. Electronic medical record data to identify variables associated with a fibromyalgia diagnosis: importance of health care resource utilization. J Pain Res. 2015;8: 131-8. doi:10.2147/JPR.S74900. PubMed PMID: 25784819; PubMed Central PMCID: PMCPMC4356680

60. Di Franco M, lannuccelli C, Bazzichi L, Atzeni F, Consensi A, Salaffi F, et al. Misdiagnosis in fibromyalgia: a multicentre study. Clin Exp Rheumatol. 2011; 29(6 Suppl 69):S104-8. PubMed PMID: 22243557

\section{Submit your next manuscript to BioMed Central and we will help you at every step:}

- We accept pre-submission inquiries

- Our selector tool helps you to find the most relevant journal

- We provide round the clock customer support

- Convenient online submission

- Thorough peer review

- Inclusion in PubMed and all major indexing services

- Maximum visibility for your research

Submit your manuscript at www.biomedcentral.com/submit 